\title{
Improved Finite Control Set Optimal Control for PMSM in Rocket Launcher Servo System
}

\author{
Di-fen Shi, Yuan-long Hou (D, Xiao-hui Gu, and Run-min Hou \\ School of Mechanical Engineering, Nanjing University of Science and Technology, Nanjing 210094, China \\ Correspondence should be addressed to Yuan-long Hou; houyuanlong1964@163.com and Run-min Hou; 187189579@qq.com
}

Received 26 December 2019; Revised 25 March 2020; Accepted 9 May 2020; Published 7 August 2020

Academic Editor: Angelo Marcelo Tusset

Copyright ( $\odot 2020$ Di-fen Shi et al. This is an open access article distributed under the Creative Commons Attribution License, which permits unrestricted use, distribution, and reproduction in any medium, provided the original work is properly cited.

\begin{abstract}
Permanent magnet synchronous motors (PMSMs) have been widely applied in the shipborne rocket launcher systems due to their high torque density and high efficiency. However, since there are many external disturbances from the shipborne rocket launcher, the tracking performance under random noises needs to be improved. In this paper, an improved finite control set optimal control (IFCSOC) based on a super-twisting extended state observer (SESO) is investigated for position tracking control of PMSMs. The SESO can improve the anti-interference ability of the proposed controller. Moreover, in order to improve tracking accuracy, Taylor's formula is used to solve the phase-lag problem of nonlinear tracking differentiator in IFCSOC. Simulation shows that compared with conventional FCSOC, IFCSOC exhibits better robustness under random disturbances. Furthermore, the semiphysical experiment is conducted to verify the proposed IFCSOC strategy.
\end{abstract}

\section{Introduction}

Permanent magnet synchronous motors (PMSMs) are commonly used in shipborne rocket launcher systems for attitude adjustment distinguished by high torque density and high efficiency. Under the complex marine conditions, the shipborne rocket launcher system is easy to be disturbed and tends to exhibit a nonlinear behavior, which requires the controller to possess strong robustness to the rapidly changing disturbances.

Various control methods have been studied for nonlinear systems due to their prominent control performance [1-7]. A sampled-data output feedback controller design technique is investigated in [4], targeting for nonlinear systems with uncontrollable and unobservable linearizations around the origin. Nevertheless, it is not easy to determine whether the wave fluctuation that imposed on the shipborne rocket launcher system satisfies the assumption of such design technique.

Finite control set optimal control (FCSOC) is a new control method receiving more and more attention, it can be applied to systems with nonlinearities and predigest control process $[5,6]$. However, to gain high performance, the FCSOC strategy needs an accurate model of the system.
Considering the application in the shipborne rocket launcher, the rapidly changing disturbances from marine environment will to large extent cause performance degradation. To address this and improve the system robustness, the external disturbances must be well estimated for compensation.

Active disturbance rejection control (ADRC) is marked by strong robustness. However, when applied to the highorder process, such design technique and the associated parameter tuning will be complicated. Also, it tends to have the slow convergence speed since it mainly focuses on the low-frequency disturbance [7]. To overcome this and gain better anti-interference ability, sliding-mode active disturbance rejection control (SADRC) is proposed in [7], where the high-order sliding-mode technology is used to construct the estimator for internal coupling factors and external disturbances. In [8], it is combined with a nonlinear extended state observer (NESO) to realize the position loop control of the PMSM. Nevertheless, there still exist two challenges. On the one hand, applying the sliding-mode control technique brings the chattering phenomenon, this requires for estimators with fast convergence algorithms. On the other hand, the phase lag still exists when tracking the reference signals due to the discrete form of nonlinear 
tracking differentiator (DNTD). To reduce the phase lags, a strict proof for some specific conditions of nonlinear tracking differentiator (NTD) is made by Guo and Zhao [9]. The research studies of the convergence, rapidity improvement and its high-order form application are carried out in [10-13]. Cataloging former references, Jinping Feng proposes a Taylor's formula-based phase compensation method to decrease phase lags [14].

The disturbance estimator is the critical part of ADRC, which is used to compensate the difference between the real model and the nominal model caused by external disturbances and changes of model parameters. There are various disturbance estimators, among which the ESO is one of the most widely used. It is because it requires the least mathematical model information and can describe the internal and external disturbances together [15-17]. The ESO is used to estimate rapidly changing disturbances in [18] and used to estimate the system disturbances for feedforward compensation in the proposed composite continuous fast terminal sliding-mode control (CFTSMC) [19]. For nonlinear systems, the convergence of ESO will be challenged. A practical way is to combine it with the super-twisting algorithm, forming the so-called super-twisting ESO (SESO). The SESO can achieve a better estimation performance as it can achieve finite-time convergence. A longitudinal study of the modified super-twisting algorithm is used to estimate state variables and disturbances in [20]. A SESO is studied in [21] with an adaptive strategy to estimate various disturbances for a diesel engine air path. These research studies show that the SESO exhibits a higher convergence speed than the general ESO for nonlinear systems.

This paper proposes the IFCSOC strategy to gain better robustness and tracking accuracy for the PMSM system in the shipborne rocket launcher. In the proposed control strategy, the adaptive disturbance compensation FCSOC strategy is applied for the current loop control, where the SESO is used to achieve finite-time stability. Also, SADRC is combined with Taylor's formula for the position loop control, reducing the phase lags resulting from the DNTD. Finally, simulation and semiphysical experiments are carried out for validation.

\section{Model Description}

A surface-mounted PMSM is used in this shipborne rocket launcher system. In the $d$-q coordinate system, the general model is

$$
\left\{\begin{array}{l}
\dot{i}_{d}=-\frac{1}{L_{d}}\left(R i_{d}+L_{q} p i_{q} \omega_{r}+u_{d}\right)+f_{d}, \\
\dot{i}_{q}=-\frac{1}{L_{q}}\left(R i_{q}+L_{d} p i_{d} \omega_{r}-\psi_{f} p \omega_{r}+u_{q}\right)+f_{q}, \\
\dot{\omega}_{r}=\frac{1}{J}\left(T_{e}-T_{L}-B \omega_{r}\right),
\end{array}\right.
$$

where $u_{d}$ and $u_{q}$ are stator voltages; $i_{d}$ and $i_{q}$ are stator currents; $L_{d}$ and $L_{q}$ are stator inductances; $\psi_{f}$ is magnetic flux; $R$ is stator resistance; $p$ is pole-pair numbers in the PMSM; $J$ is inertia moment; $B$ is friction modulus; $\omega_{r}$ is angular velocity of the rotor; $T_{e}$ is electromagnetic torque; $T_{L}$ is load torque.

To simplify the mathematical expression, we define

$$
\left\{\begin{array}{l}
X_{1}=\left[i_{d}, i_{q}\right]^{T} ; \\
X_{2}=F=\left[f_{d}, f_{q}\right]^{T}, \\
\dot{F}=\vartheta=\left[\vartheta_{1}, \vartheta_{2}\right]^{T}, \\
U=\left[u_{d}, u_{q}\right]^{T}, \\
H=\left[L p i_{q} \omega_{r}, L p i_{d} \omega_{r}-\psi_{f} p \omega_{r}\right]^{T}, \\
Y=X_{1},
\end{array}\right.
$$

where $\max \left[\vartheta_{1}, \vartheta_{2}\right]^{T}<l$. Subsequently, by substituting equation (2) into equation (1), we obtain

$$
\left\{\begin{array}{l}
\dot{X}_{1}=\frac{1}{L}\left(-R X_{1}+H+U\right)+X_{2}, \\
\dot{X}_{2}=\vartheta .
\end{array}\right.
$$

2.1. Current Loop FCSOC Controller of PMSM. The overall control diagram of the PMSM servo system is shown in Figure 1. It can be seen that the SESO and FCSOC are applied in the current control loop. The SESO is an ESO characterized with high-precision observation in changing disturbance and states with the super-twisting algorithm, which can improve the anti-interference ability of IFCSOC. When disturbance exists, the adaptive disturbance compensation FCSOC performs better on dynamic response.

2.2. Design of the SESO. The SESO is a general ESO combined with the super-twisting algorithm; the observation of ESO is

$$
\left\{\begin{array}{l}
Z_{1}=\left[i_{d}^{*}, i_{q}^{*}\right]^{T}, \\
Z_{2}=\left[f_{d}^{*}, f_{q}^{*}\right]^{T}, \\
\bar{I}=X_{1}-Z_{1}, \\
\bar{F}=X_{2}-Z_{2}, \\
\sigma_{1}=\bar{I}+\frac{R}{L} \bar{I}+q|\bar{I}|^{\lambda} \operatorname{sgn}(\bar{I}),
\end{array}\right.
$$

where $Z_{1}$ is the observation of $X_{1}, Z_{2}$ is the estimation of disturbance $F, \bar{I}$ and $\bar{F}$ are estimations of transient errors, $\sigma_{1}$ is a variable in sliding-mode control, and $\lambda$ is a constant $(1 / 2<\lambda<1)$.

To simplify the mathematical expression, the following notions are claimed: 


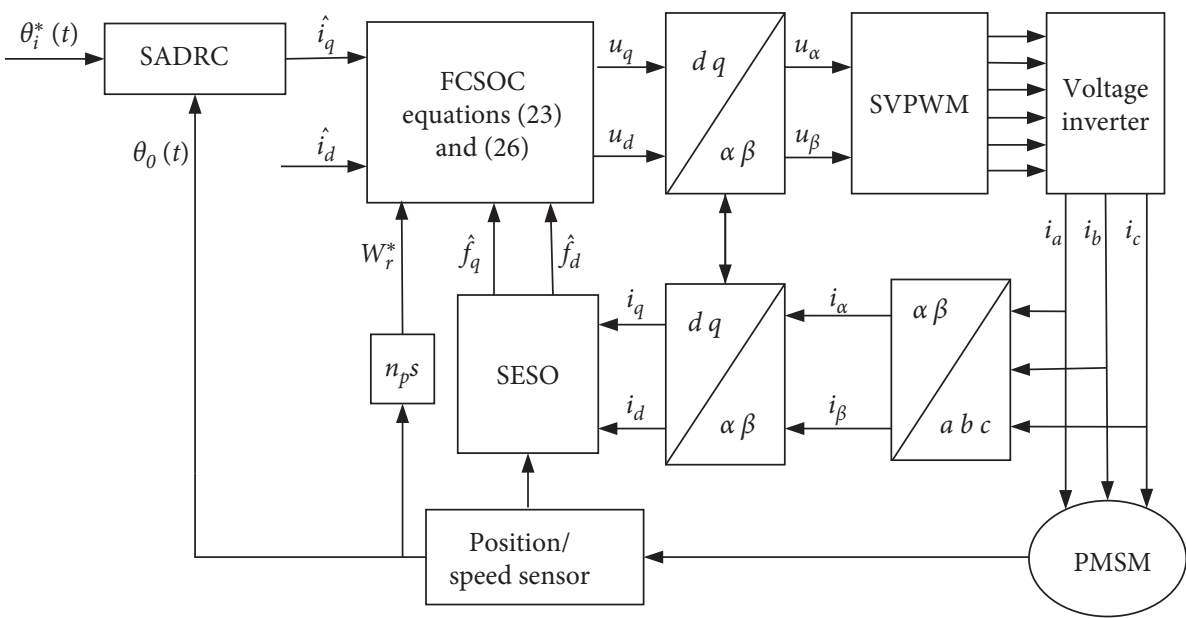

FIgURE 1: Diagram of a PMSM control system.

$\operatorname{sgn}(w)^{\rho}=\left[\left|w_{1}\right|^{\rho_{1}} \operatorname{sgn}\left(w_{1}\right), \ldots,\left|w_{n}\right|^{\rho_{n}} \operatorname{sgn}\left(w_{n}\right)\right]^{T}$,

$\mu \operatorname{sgn}(w)^{\rho}=\left[\left|\mu_{1}\right|^{\rho_{1}} \operatorname{sgn}\left(w_{1}\right), \ldots,\left|\mu_{n}\right|^{\rho_{n}} \operatorname{sgn}\left(w_{n}\right)\right]^{T}$.

The SESO is constructed as

$$
\left\{\begin{array}{l}
\dot{Z}_{1}=\frac{1}{L}\left(-R X_{1}+H+U\right)+Z_{2}-\delta_{1} \Theta_{1}(\bar{I}), \\
\dot{Z}_{2}=\kappa(\bar{I}) \dot{\bar{I}}-\delta_{2} \Theta_{2}(\bar{I}),
\end{array}\right.
$$

where

$$
\begin{aligned}
\Theta_{1}(\bar{I}) & =|\bar{I}|^{(1 / 2)} \operatorname{sign}(\bar{I})+\bar{I}, \\
\Theta_{2}(\bar{I}) & =\frac{1}{2} \operatorname{sgn}(\bar{I})+\bar{I}+\frac{3}{2}|\bar{I}|^{(1 / 2)} \operatorname{sgn}(\bar{I}), \\
\kappa(\bar{I}) & =(\lambda+1) q|\bar{I}|^{\lambda} \operatorname{sign}(\bar{I}) .
\end{aligned}
$$

Note that $\delta_{1}$ and $\delta_{2}$ are two parameters to be adjusted. According to (3) and (6)fd6, the corresponding errors in observation of the SESO (6) can be expressed as follows:

$$
\left\{\begin{array}{l}
\dot{\bar{I}}=\bar{F}-\delta_{1} \Theta_{1}(\bar{I}), \\
\dot{\bar{F}}=\kappa(\bar{I}) \dot{\bar{I}}-\delta_{2} \Theta_{2}(\bar{I}) .
\end{array}\right.
$$

Assume that $\bar{I} \neq[0,0]^{T}$ in the initial state. Set $V_{0}$ as Lyapunov function:

$$
V_{0}(\vartheta(t))=\vartheta(t)^{T} P \vartheta(t),
$$

where $\vartheta(t)^{T}=\left[\Theta_{1}(\bar{I}), \bar{F}\right]$, in which $\dot{\vartheta}(t)$ is expressed as follows:

$$
\dot{\vartheta}(t)=\Theta_{1}^{\prime}(\bar{I})\left[\begin{array}{c}
-\delta_{1} \Theta_{1}(\bar{I})+\bar{F} \\
-\delta_{2} \Theta_{2}(\bar{I})+\frac{\kappa(\bar{I}) \dot{\bar{I}}}{\Theta_{1}^{\prime}(\bar{I})}
\end{array}\right]=\Theta_{1}^{\prime}[A \vartheta(t)+B \varepsilon(t)],
$$

where $\quad \varepsilon(t)=\left((\kappa(\bar{I}) \dot{\bar{I}}) / \Theta_{1}^{\prime}(\bar{I})\right), \quad A=\left[\begin{array}{ll}-\delta_{1} & 1 \\ -\delta_{2} & 0\end{array}\right], \quad \Theta_{1}^{\prime}(\bar{I})=$ $\left(\left(\bar{I}^{-(1 / 2)}\right) / 2\right)+1$, and $B=\left[\begin{array}{l}0 \\ 1\end{array}\right]$.

Assumption 1 (see [22]). The disturbance $\varepsilon(t)$ and $\vartheta(t) \in R^{2}$ satisfy the following inequality:

$$
\Lambda(\vartheta(t), \varepsilon(t))=\left[\begin{array}{ll}
\vartheta(t) & \varepsilon(t)
\end{array}\right]\left[\begin{array}{cc}
H & S^{T} \\
S & -R
\end{array}\right]\left[\begin{array}{l}
\vartheta(t) \\
\varepsilon(t)
\end{array}\right] \geq 0,
$$

where $H \geq 0, R \geq 0, S=\left[\begin{array}{ll}c & m\end{array}\right]$, and $c$ and $m$ are two constants.

The convergence time of SESO in equation (8) can be determined in finite time; for the details, refer to the following theorem.

Theorem 1 (see [23]). If the following inequality holds,

$$
\left[\begin{array}{cc}
A^{T} P+P A+\tau P+H & P B+S^{T} \\
B^{T} P+S & -R
\end{array}\right] \leq 0,
$$

the allowable convergence time can be represented as

$$
T_{s}=\frac{2}{\tau} \ln \left(\frac{2}{\sqrt{\lambda_{\min }}\{P\}} V_{0}^{(1 / 2)}(\vartheta(0))+1\right),
$$

where $\tau$ and $R$ are positive constants and $P$ is a positive and symmetric matrix. In this condition, the observation of SESO in (8) can realize finite-time stability; $\vartheta(0)$ is the initial condition.

2.3. Disturbance Compensation FCSOC for PMSM. The disturbance estimation is performed in the previous section. FCSOC is adopted to track the reference current $\widehat{i}_{d}$ and $\widehat{i}_{q}$. The current errors can be expressed as

$$
\left[\begin{array}{c}
\dot{e}_{d} \\
\dot{e}_{q}
\end{array}\right]=\left[\begin{array}{c}
\hat{i}_{d}-i_{d} \\
\hat{i}_{q}-i_{q}
\end{array}\right] .
$$


The differentiation of equation (14) is

$\left[\begin{array}{c}\dot{e}_{d} \\ \dot{e}_{q}\end{array}\right]=\left[\begin{array}{c}\frac{\widehat{\mathrm{d}} \hat{i}_{d}}{\mathrm{~d} t}-\frac{1}{L}\left(-R i_{d}+L p i_{q} w_{r}+u_{d}\right)-f_{d} \\ \frac{\widehat{\mathrm{d}} \hat{i}_{q}}{\mathrm{~d} t}-\frac{1}{L}\left(-R i_{d}+L p i_{d} w_{r}-\psi_{f} p w_{r}+u_{q}\right)-f_{q}\end{array}\right]$.

To compensate the disturbances (which has been estimated before), the control law is

$$
\left[\begin{array}{c}
u_{d}^{*} \\
u_{q}^{*}
\end{array}\right]=\left[\begin{array}{c}
L \frac{\widehat{d} \hat{i}_{d}}{\mathrm{~d} t}+R i_{d}-L p i_{q} w_{r}+k_{1}\left|e_{d}\right|^{\eta} \operatorname{sgn}\left(e_{d}\right)-L \widehat{f}_{d} \\
d \hat{\mathrm{i}}_{q} \\
\mathrm{~d} t
\end{array}\right.
$$

Substituted by equation (16), equation (15) is transformed as

$$
\left[\begin{array}{c}
\dot{e}_{d} \\
\dot{e}_{q}
\end{array}\right]=\left[\begin{array}{c}
-\widetilde{f}_{d}-k_{1}\left|e_{d}\right|^{\eta} \operatorname{sgn}\left(\frac{e_{d}}{L}\right) \\
-\tilde{f}_{q}-k_{2}\left|e_{q}\right|^{\eta} \operatorname{sgn}\left(\frac{e_{q}}{L}\right)
\end{array}\right],
$$

where $\tilde{f}_{d}$ and $\tilde{f}_{q}$ are estimation errors:

$$
\left[\begin{array}{c}
\tilde{f}_{d} \\
\tilde{f}_{q}
\end{array}\right]=\left[\begin{array}{c}
f_{d}-\hat{f}_{d} \\
f_{q}-\hat{f}_{q}
\end{array}\right] .
$$

Set Lyapunov function $V_{1}$ as

$$
V_{1}=e_{d}^{2}+e_{q}^{2}+\frac{1}{2 \gamma_{1}} \tilde{f}_{d}^{2}+\frac{1}{2 \gamma_{2}} \tilde{f}_{q}^{2},
$$

where $\gamma_{2}$ and $\gamma_{3}$ are positive parameters.

The derivative of equation (19) is

$$
\begin{aligned}
\dot{V}_{1}= & e_{d} \dot{e}_{d}+e_{q} \dot{e}_{q}+\frac{1}{\gamma_{1}} \tilde{f}_{d} \dot{\tilde{f}}_{d}+\frac{1}{\gamma_{2}} \tilde{f}_{q} \dot{\tilde{f}}_{q} \\
= & e_{d}\left(-\tilde{f}_{d}-k_{1}\left|e_{d}\right|^{\eta} \operatorname{sgn}\left(\frac{e_{d}}{L}\right)\right) \\
& +e_{q}\left(-\tilde{f}_{q}-k_{2}\left|e_{q}\right|^{\eta} \operatorname{sgn}\left(\frac{e_{q}}{L}\right)\right)+\frac{1}{\gamma_{1}} \tilde{f}_{d} \dot{\tilde{f}}_{d}+\frac{1}{\gamma_{2}} \widetilde{f}_{q} \dot{\tilde{f}}_{q} \\
= & \frac{-k_{1}}{L\left|e_{d}\right|^{\eta+1}}-\frac{k_{2}}{L\left|e_{q}\right|^{\eta+1}}+\tilde{f}_{d}\left(\frac{1}{\gamma_{1}} \tilde{\tilde{f}}_{d}-e_{d}\right) \\
& +\tilde{f}_{q}\left(\frac{1}{\gamma_{2}} \dot{\tilde{f}}_{q}-e_{q}\right) .
\end{aligned}
$$

To compensate disturbances, control laws are given as

$$
\left\{\begin{array}{l}
\dot{\tilde{f}}_{d}=\gamma_{1} e_{d} \\
\dot{\tilde{f}}_{q}=\gamma_{2} e_{q} .
\end{array}\right.
$$

By using of the adaptive law in equation (21), the estimation of $\widetilde{f}_{d}$ and $\widetilde{f}_{q}$ can be more accurate.

\section{The SADRC Controller in Position Loop}

3.1. The Structure of SADRC Controller. In the overall IFCSOC system, a SADRC controller is adopted in position control. The details are shown in Figure 2. Generally, considering $i_{d}^{*}=0$ in a synchronously rotating coordinate system, the position loop is expressed as

$$
\frac{\mathrm{d}^{2} \theta_{i}(t)}{\mathrm{d} t^{2}}=-\frac{B}{J} \dot{\theta}_{i}(t)+\frac{1.5 n_{p} \psi_{f}}{J} i_{q}^{*}-\frac{T_{L}}{J},
$$

where $i_{q}^{*}$ and $i_{q}$ are the reference current and real current of the stator in the $q$ coordinate axis, respectively; $\theta_{i}(t)$ is the rotor position; $T_{L}$ is the load torque; $\psi_{f}$ is the rotor flux; $n_{p}$ is the number of pole pairs; $J$ is the inertia of the rotor; $B$ is the friction parameter.

Set new state variables as $x_{1}=\theta_{i}(t), x_{2}=\left(\left(\mathrm{d} \theta_{i}(t)\right) / \mathrm{d} t\right)=$ $\omega, u=i_{q}, \quad b=\left(1.5 n_{p .} \psi_{f} / J\right), \quad f_{1}\left(x_{1}, x_{2}, t\right)=\left(-T_{L} / J\right)$, and $f_{0}\left(x_{1}, x_{2}\right)=-(B / J) \theta_{i}(t)$. The mathematical model of position loop can be expressed as

$$
\begin{aligned}
& \dot{x}_{1}=x_{2}=\frac{\mathrm{d} \theta_{i}(t)}{\mathrm{d} t}, \\
& \dot{x}_{2}=\frac{\mathrm{d}^{2} \theta_{i}(t)}{\mathrm{d} t^{2}}=f_{0}\left(x_{1}, x_{2}\right)+f_{1}\left(x_{1}, x_{2}, t\right)+b u, \\
& g=x_{1}=\frac{\mathrm{d} \theta_{i}(t)}{\mathrm{d} t},
\end{aligned}
$$

where $x_{1}$ is the tracking signal of $\theta_{i}^{*} ; x_{2}$ is the differentiation of $x_{1} ; f_{0}\left(x_{1}, x_{2}\right)$ is the known disturbance; $f_{1}\left(x_{1}, x_{2}, t\right)$ is the unknown external disturbance; $\omega(t)=f_{0}\left(x_{1}\right.$, $\left.x_{2}\right)+f_{1}\left(x_{1}, x_{2}, t\right)$ is the total disturbance.

In the SADRC controller, the nonlinear tracking differentiator (NTD) is used to track state variables $x_{1}$ and $x_{2}$. $z_{1}, z_{2}$, and $z_{3}$ are the system state values and disturbance estimated by using the ESO. $e_{1}=x_{1}-z_{1}$ represents the tracking error. The integral and differentiation of tracking errors are $e_{0}=\int e_{1} \mathrm{~d} t$ and $e_{2}=x_{2}-z_{2}$. From Figure 2, we can obtain reference current in the following form:

$$
\begin{aligned}
i_{q}^{*}= & \beta_{0} \mathrm{fal}\left(e_{0}, \alpha_{5}, \delta_{4}\right)+\beta_{1} \mathrm{fal}\left(e_{1}, \alpha_{1}, \delta_{1}\right)+\beta_{2} \mathrm{fal}\left(e_{2}, \alpha_{2}, \delta_{2}\right) \\
& -\frac{z_{3}+f_{0}\left(z_{1}, z_{2}\right)}{b}
\end{aligned}
$$

where $\beta_{0}, \beta_{1}$, and $\beta_{2}$ are the $e_{0}, e_{1}$, and $e_{2}$ gains of NLSEF, respectively. The nonlinear function fal $(e(k), \alpha, \delta)$ can be defined as 


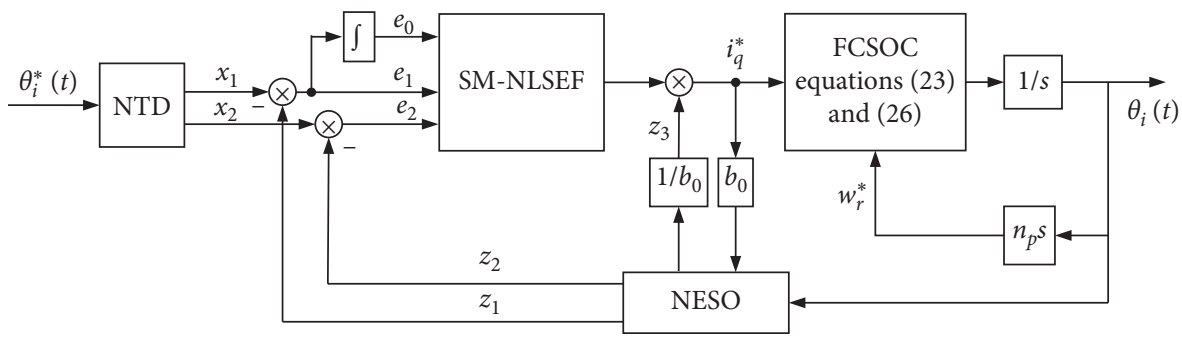

FIGURE 2: Structure of the SADRC-FCSOC controller.

$$
\mathrm{fal}(e(k), \alpha, \delta)= \begin{cases}|e(k)|^{\alpha} \operatorname{sgn}(e(k)), & |e(k)|>\delta, \\ \frac{e(k)}{\delta^{1-\alpha},} & |e(k)| \leq \delta .\end{cases}
$$

3.2. Taylor's Formula Compensation for the Nonlinear Tracking Differentiator (NTD). The nonlinear tracking differentiator (NTD) in ADRC is used to track reference signal and derivative of the reference signal. The NTD process can be given as

$$
\begin{aligned}
& x_{1}(k+1)=x_{1}(k)+h x_{2}(k), \\
& x_{2}(k+1)=x_{2}(k)+h f s t\left(x_{1}(k)-g_{d}(k), x_{2}(k), r, h_{0}\right),
\end{aligned}
$$

where $g_{d}(k)$ is the desired trajectory; $h_{0}$ is the filter coefficient; $r$ is the speed coefficient; $h$ is the sampling step; $x_{1}(k)$ and $x_{2}(k)$ are the tracking signal of $g_{d}(k)$ and $\dot{g}_{d}(k)$.

Generally, tracking reference can be improved by raising rand $h_{0}$. The definition of nonlinear function $f s t(\cdot)$ is

$$
\begin{aligned}
f_{s t}\left(x_{1}(k), x_{2}(k), r, h\right) & = \begin{cases}-r\left(\frac{a}{d}\right), & |a| \leq d, \\
-r \operatorname{sgn}(a), & |a|>d,\end{cases} \\
a & = \begin{cases}x_{2}(k)+\frac{a_{0}-d}{2} \operatorname{sgn}(y(k)), & |y(k)|>d_{0}, \\
x_{2}(k)+\frac{y(k)}{h}, & |y(k)| \leq d_{0},\end{cases} \\
d & =r h, \\
d_{0} & =d h, \\
a_{0} & =\sqrt{d^{2}+8 r|y(k)|,} \\
g(k) & =x_{1}(k)+h x_{2}(k) .
\end{aligned}
$$

According to Taylor's formula, the value of $g_{d}(k)$ at $k+$ $\Delta k$ can be predicted as

$$
\begin{aligned}
g_{d}(k+\Delta k)= & g_{d}(k)+\frac{g_{d}^{\prime}(k)}{1 !} \Delta k+\frac{g_{d}^{\prime \prime}(k)}{2 !}(\Delta k)^{2} \\
& +\cdots+\frac{g_{d}^{(n)}(k)}{n !}(\Delta k)^{n}+\frac{g_{d}^{(n+1)}(\xi)}{(n+1) !}(\Delta k)^{n+1} .
\end{aligned}
$$

In Figure $3, g_{d}(k)$ is the true signal and $x_{1}(k)$ is the tracking signal, we can see that there is a phase delay between the tracking signal and the true signal. Phase delay happens because the tracking speed is not appropriate all the time. From Figure 3 , the true signal $g_{d}\left(k_{1}\right)$ equals the tracking signal $x_{1}\left(k_{2}\right)$. Similarly, the true signal at time $k_{n}$ equals the tracking signal at $k_{n+1}$, the delay time $\Delta k=k_{n+1}-k_{n}$. The value of tracking signal may be smaller or larger than that of true signal. For example, $x_{1}\left(k_{1}\right)$ is larger than $g_{d}\left(k_{1}\right)$, while $x_{1}\left(k_{n}\right)$ is smaller than $g_{d}\left(k_{n}\right)$. Therefore, Taylor's formula is used in the DNTD to compensate the phase delay; the method can be expressed as

$$
\begin{aligned}
\tilde{g}_{d}\left(k_{n+1}\right)= & g_{d}\left(k_{n}\right)+\frac{g_{d}^{\prime}\left(k_{n}\right)}{1 !}\left(k_{n+1}-k_{n}\right) \\
& +\frac{g_{d}^{\prime \prime}\left(k_{n}\right)}{2 !}\left(k_{n+1}-k_{n}\right)^{2}+\cdots+\frac{g_{d}^{(n)}\left(k_{n}\right)}{n !}\left(k_{n+1}-k_{n}\right)^{n} \\
& +\frac{g_{d}^{(n+1)}(\xi)}{(n+1) !}\left(k_{n+1}-k_{n}\right)^{n+1} .
\end{aligned}
$$

If we obtain the delay time $\Delta k=k_{n+1}-k_{n}=r_{C} h$, we can make use of Taylor's formula to compensate retardation for accuracy tracking. In this paper, we set the compensate step as $r_{C}$. Then, equation (29) can be rewritten as

$$
\begin{aligned}
\tilde{g}_{d}\left(k_{n+1}\right)= & x_{1}\left(k_{n+1}\right)+\frac{x_{2}\left(k_{n+1}\right)}{1 !}\left(k_{n+1}-k_{n}\right) \\
& +\frac{x_{3}\left(k_{n+1}\right)}{2 !}\left(k_{n+1}-k_{n}\right)^{2}+\cdots+\frac{x_{n}\left(k_{n+1}\right)}{n !}\left(k_{n+1}-k_{n}\right)^{n} .
\end{aligned}
$$

Taylor's approach is expected to compensate phase delay based on the aforementioned theory; the method in [24] is given as

$$
g_{d}\left(k_{n+1}\right)=x_{1}\left(k_{n+1}\right)+r_{C} \Delta k \cdot x_{2}\left(k_{n+1}\right) .
$$

Subsequently, equation (31) can be expressed as

$$
\begin{aligned}
g(k+1) & =x_{1}(k+1)+r_{C} \Delta k \cdot x_{2}(k+1), \\
x_{2}(k+1) & =x_{2}(k)+h f s t\left(x_{1}(k)-g_{d}(k), x_{2}(k), r, h_{0}\right) .
\end{aligned}
$$

Set $r_{C}$ as the time step in compensation; $M$ is the maximum value of $\left|f^{(n+1)}(\xi)\right|$ in $\left[k_{n}, k_{n+1}\right]$, and the Lagrange 


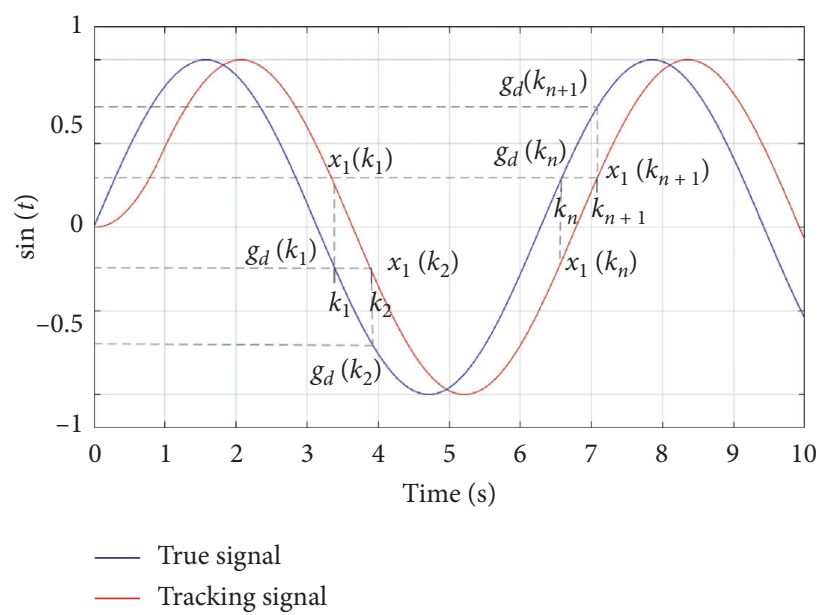

FIgURE 3: The schematic diagram of phase delay.

remainder $R_{n}$ of the proposed Taylor's approach should meet

$$
R_{n} \leq \frac{M}{(n+1) !}\left|x-x_{0}\right|^{n+1} .
$$

3.3. Nonlinear Extended State Observer (NESO). NESO can estimate disturbances quickly and accurately. Therefore, this paper combines the disturbance observer (DOB) with the ESO to derive the NESO. Let $x_{1}=\theta_{i}(t)$ and $x_{2}=\left(\mathrm{d} \theta_{i}(t) / \mathrm{d} t\right)=\omega$, then

$$
\left\{\begin{array}{l}
\dot{x}_{1}=x_{2}=\frac{\mathrm{d} \theta_{i}(t)}{\mathrm{d} t} \\
\dot{x}_{2}=\frac{\mathrm{d}^{2} \theta_{i}(t)}{\mathrm{d} t^{2}}=f_{0}\left(x_{1}, x_{2}\right)+f_{1}\left(x_{1}, x_{2}, t\right)+b u=f\left(x_{1}, x_{2}, t\right)+b i_{q} .
\end{array}\right.
$$

Choose a new state as

$$
\left\{\begin{array}{l}
x_{2}=f\left(x_{1}, x_{2}, t\right), \\
\dot{x}_{2}=L(t),
\end{array}\right.
$$

with $L(t)=\dot{f}\left(x_{1}, x_{2}, t\right)$.

The NESO in ADRC is designed to estimate all the state variables and disturbances.

$$
\begin{aligned}
e(k) & =z_{1}(k)-y(k), \\
z_{1}(k+1) & =z_{1}(k)-h\left[z_{2}(k)-\beta_{01} e(k)\right], \\
z_{2}(k+1) & =z_{3}(k)-\beta_{02} \mathrm{fal}\left(e(k), \alpha_{1}, \delta_{1}\right)+g i_{q}, \\
\dot{z}_{3}(k+1) & =-l z_{3}(k)-l\left[l w_{r}+f(x)+g i_{q}\right], \\
\widehat{f} & =z_{3}(k)+l w_{r},
\end{aligned}
$$

where $f(x)=-(B / J) w_{r}, g(x)=\left(3 n_{p} \varphi_{f} / 2 J\right)$, and $l$ is the gain matrix of NESO.

3.4. Sliding-Mode Nonlinear State Error Feedback (SM$N L S E F)$. To improve the response speed and simplify the
TABle 1: Parameters in the PMSM.

\begin{tabular}{lc}
\hline Parameter & Value \\
\hline Load moment of inertia $(J)$ & $5.556 \times 10^{-3} \mathrm{~kg} \times \mathrm{m}^{2}$ \\
Friction coefficient $(B)$ & $1.43 \times 10^{-4}(\mathrm{~N} \times \mathrm{m}) /\left(\mathrm{rad}^{-1}\right)$ \\
Load disturbing moment $\left(T_{L}\right)$ & $9.32 \times 10^{3} \mathrm{~kg} \times \mathrm{m}^{2}$ \\
Motor torque factor $\left(K_{t}\right)$ & $0.175(\mathrm{~N} \times \mathrm{m}) / \mathrm{A}$ \\
Reduction ratio $(i)$ & 1039 \\
Stator inductance $\left(L_{d}=L_{q}\right)$ & $21.24 \mathrm{mH}$ \\
Pole pairs $\left(n_{p}\right)$ & 3 \\
Motor magnetic flux $\left(\psi_{f}\right)$ & $0.65 \mathrm{~Wb}$ \\
\hline
\end{tabular}

parameter tuning, we select function $g\left(e_{2}(k)\right)$ instead of nonlinear operator function fal $(e(k), \alpha, \delta)$ :

$$
\left\{\begin{array}{l}
e_{1}(k+1)=x_{1}(k+1)-z_{1}(k+1), \\
e_{2}(k+1)=x_{2}(k+1)-z_{2}(k+1), \\
\dot{e}_{2}=g\left(e_{2}\right), \\
i_{q}^{*}=g\left(e_{2}\right)-z_{3} .
\end{array}\right.
$$

Design a sliding-mode surface $s(t)=c e_{2}$ and sliding function $g\left(e_{2}\right)=-k \operatorname{sgn}(s)$. According to Lyapunov stability theory, $\lim _{s \longrightarrow 0} s \dot{s}<0$ should be satisfied.

$$
s(t) \cdot \dot{s}(t)=s(t)[-k \operatorname{sgn}(s(t))]
$$

Since $k>0$ is a tunable parameter, the system is always stable. To suppress the chattering in the system and enhance the control continuity, the anti-chattering factor $G(s)$ is introduced to replace $\operatorname{sgn}(s)$ :

$$
G(s)=\frac{s}{|s|+v},
$$

where $v$ is the chattering factor, with $v>0$. The SM-NLSEF is represented as

$$
\left\{\begin{array}{l}
e_{1}(k+1)=x_{1}(k+1)-z_{1}(k+1), \\
e_{2}(k+1)=x_{2}(k+1)-z_{2}(k+1), \\
i_{q}^{*}=-k G(s)-z_{3} .
\end{array}\right.
$$




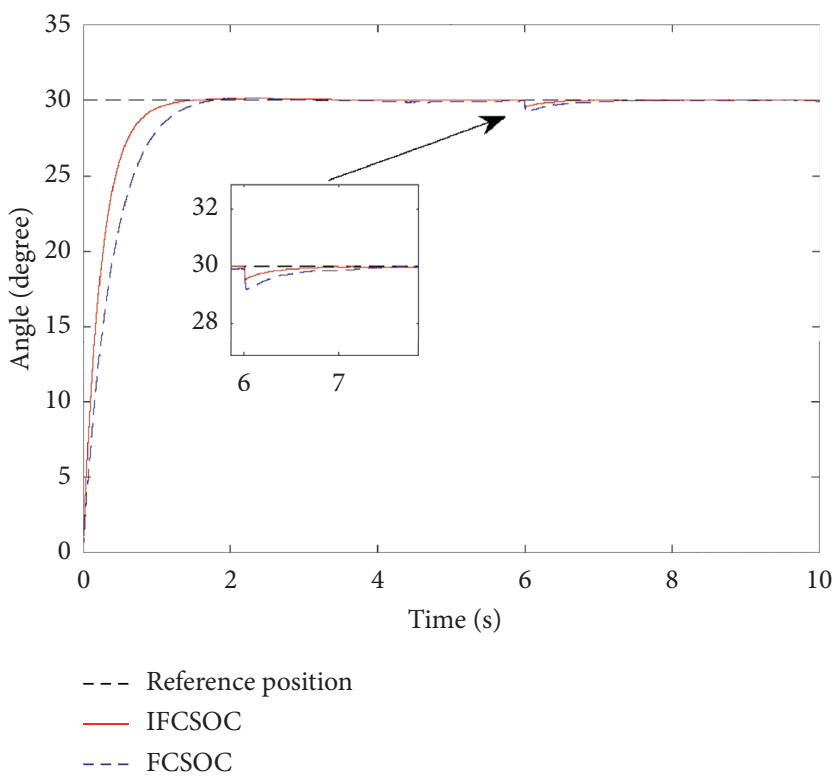

FIgURE 4: Step simulation with load step disturbance.

TABle 2: Step simulation data.

\begin{tabular}{|c|c|c|c|c|}
\hline Control strategy & Adjustment time (s) & Maximum disturbance $\left({ }^{\circ}\right)$ & Recovery time (s) & Steady-state error $\left(^{\circ}\right)$ \\
\hline IFCSOC & 1.23 & 0.34 & 0.64 & 0.032 \\
\hline FCSOC & 1.67 & 0.78 & 0.85 & 0.056 \\
\hline
\end{tabular}

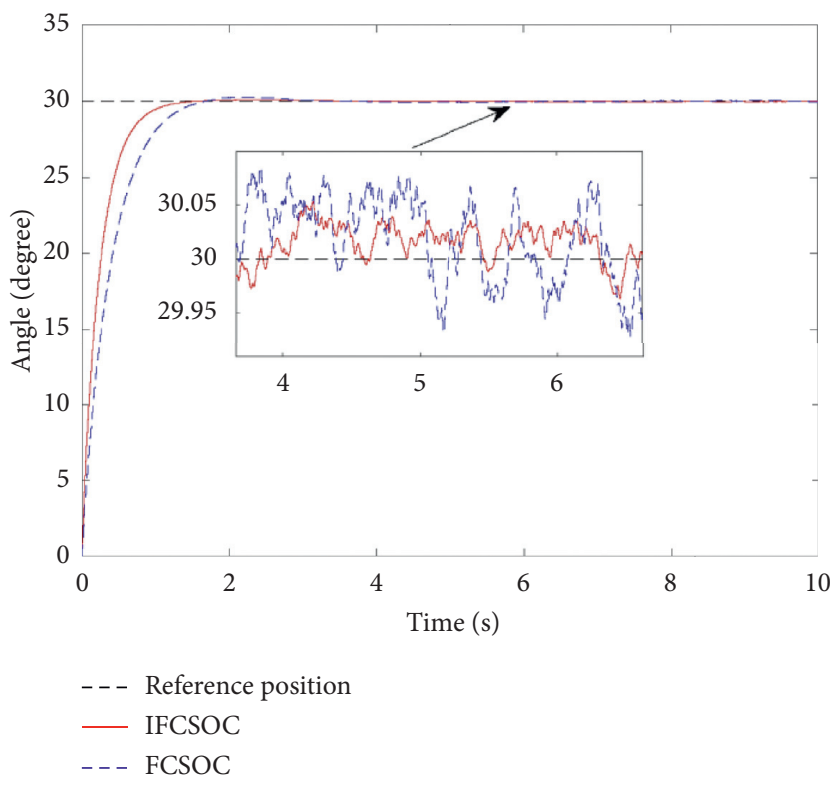

FIGURE 5: Step simulation with load rapidly changing disturbance.

3.5. Simulation and Analysis. To further explore the effectiveness of SADRC-FCSOC controller, we carry out the simulation studies of step response and sinusoidal signal tracking. The parameters of PMSM are listed in Table 1. The NTD parameters are $h=0.01$ and $r_{C}=1000$. The NESO parameters are $\beta_{01}=1$ and $\beta_{02}=15.8$. The SESO parameters 


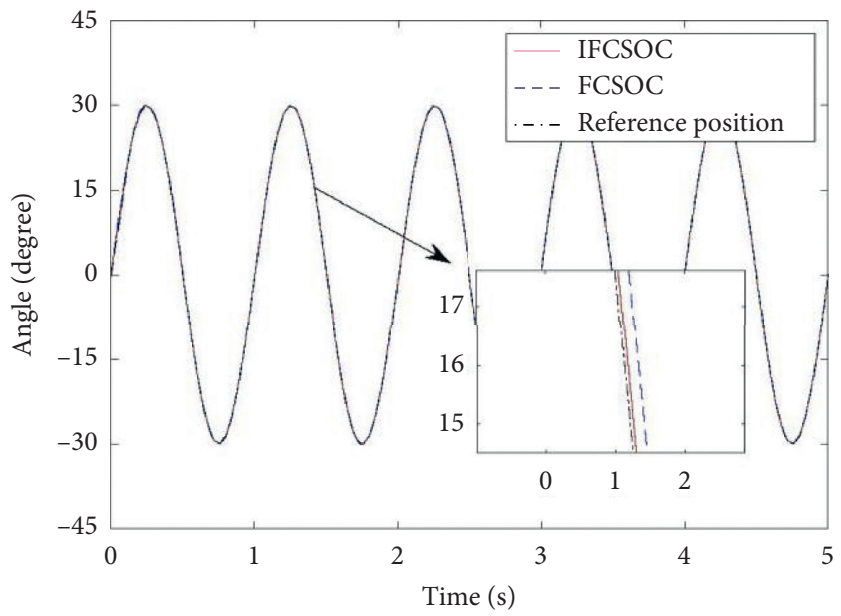

Figure 6: Tracking of the sinusoidal signal.

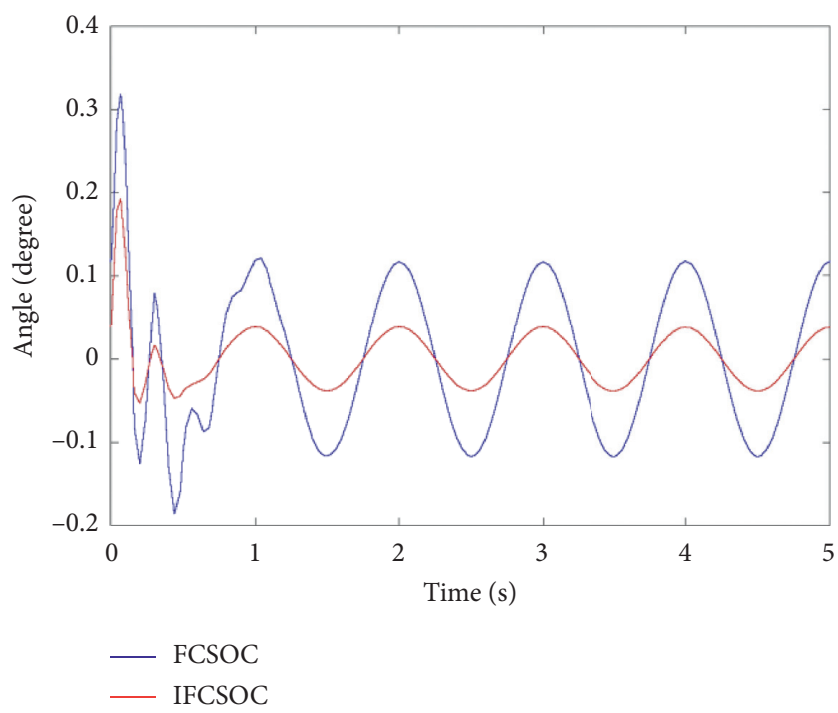

FIgURE 7: Tracking errors of the sinusoidal signal.

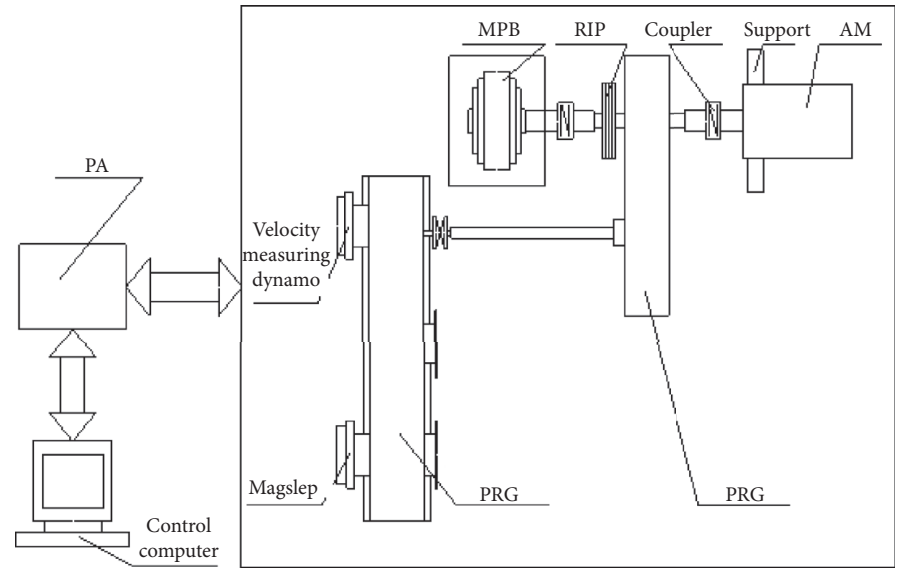

(a)

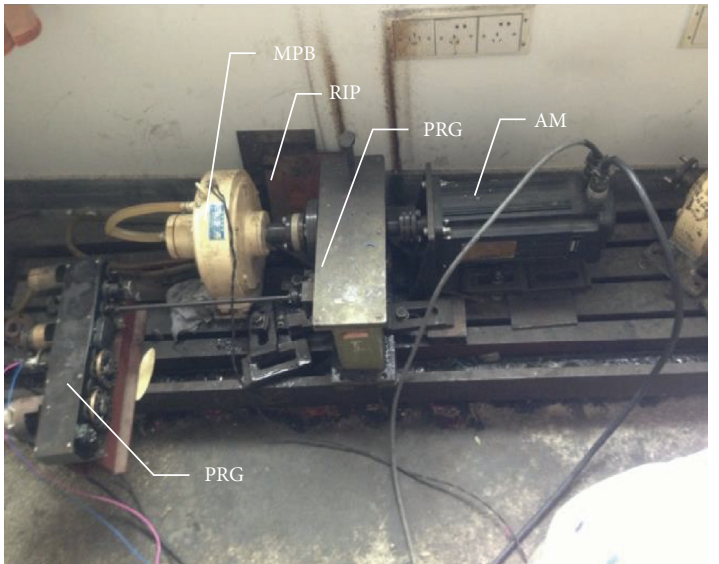

(b)

FIgURE 8: Semiphysical workbench structure. 
TABLE 3: Components of the semiphysical simulation workbench.

\begin{tabular}{lc}
\hline Abbreviation & Full name \\
\hline PA & Power amplifier \\
PRG & Precision reduction gearbox \\
LF & Loading fixture \\
RIP & Rotational inertia plate \\
MPB & Magnetic power brake \\
AM & Actuating motor \\
\hline
\end{tabular}

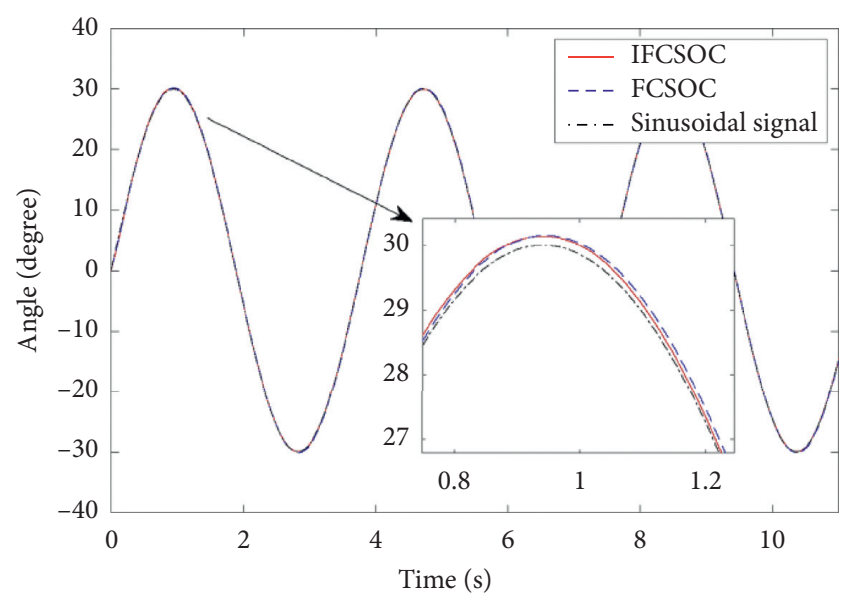

FIgURE 9: Comparison diagram of sinusoidal tracking curves.

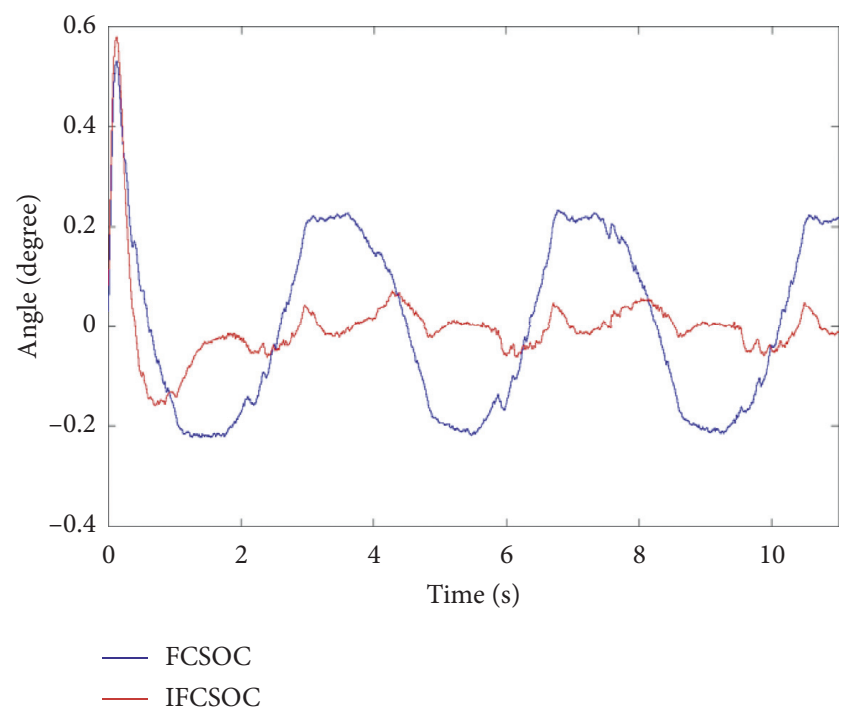

FIGURE 10: Diagram of sinusoidal tracking error curves.

are set as $\delta_{1}=\delta_{2}=0.01$. The rational parameters of FCSOC are set as $k_{1}=k_{2}=100, \eta=0.5$, and $\gamma_{1}=\gamma_{2}=3.5$.

Figure 4 shows the step response of IFCSOC and FCSOC with a $500 \mathrm{~nm}$ load added at $6.0 \mathrm{~s}$. The simulation data are recorded in Table 2 .

From Figure 4, it can be seen that IFCSOC exhibits a faster response than FCSOC. When a disturbance is added at $6.0 \mathrm{~s}$, IFCSOC has a smaller position offset response. Furthermore,
IFCSOC has better robust performance over FCSOC. The maximum disturbance value of IFCSOC is $0.34^{\circ}$ smaller than FCSOC. Also, IFCSOC has better steady-state accuracy. The steady-state error of IFCSOC is 3.2\%; meanwhile, the steadystate error of FCSOC is 5.6\%, which is 1.75 times higher than that of IFCSOC.

Figure 5 shows the step response of IFCSOC and FCSOC with rapidly changing disturbances, where the maximum steady-state errors of the IFCSOC controller and FCSOC controller are $0.049^{\circ}$ and $0.082^{\circ}$, respectively. It is indicated that the IFCSOC controller has stronger anti-interference ability than the FCSOC.

To measure the dynamic tracking performance of IFCSOC, a sinusoidal signal tracking simulation is conducted. As shown in Figure 6, IFCSOC achieves a higher accuracy compared with FCSOC in tracking the sinusoidal signal. The phase lags have been reduced when adopting the IFCSOC method, which means Taylor's formula to compensate phase delay is effective in raising tracking efficiency.

Figure 7 shows the tracking errors of sinusoidal signal, from which it can be seen that IFCSOC has a better tracking performance compared with FCSOC. The maximum tracking error of IFCSOC is $0.038^{\circ}$, while the FCSOC can reach $0.117^{\circ}$.

\section{Semiphysical Simulation Experiment}

To evaluate the performance of IFCSOC controller, a semiphysical simulation is conducted in our laboratory for position control of the PMSM. The overall view of semiphysical workbench is illustrated in Figure 8 and listed in Table 3.

The workbench is composed of the following parts: the control computer, sensor system, PA, PRG, LF, AM, RIP, and the support. The RIP and MPB are used to simulate disturbance factors in the system, such as frictional moment, load torque, and rotational inertia. By controlling the RIP, the rotational inertia can be simulated. In the same way, the MPB output torque can simulate rapidly changing torque and frictional moment.

To verify the dynamic tracking accuracy of the IFCSOC method applied in the servo system, an experiment is conducted on the semiphysical workbench for tracking a sinusoidal signal with a period of $3.768 \mathrm{~s}$ and an amplitude of $30^{\circ}$. The tracking curves of FCSOC and IFCSOC are shown in Figure 9. It can be seen that the tracking curve of IFCSOC is more accurate than that of FCSOC. Also, IFCSOC has a smaller phase delay.

Figure 10 compares the tracking errors obtained from preliminary experiments. It implies that, in the initial tracking period, the maximum tracking error of FCSOC is $0.53^{\circ}$ and IFCSOC is $0.58^{\circ}$. Entering the stabilization stage, the maximum tracking error of FCSOC is $0.203^{\circ}$ and IFCSOC is $0.073^{\circ}$. Also, it can be seen that the chattering amplitude of IFCSOC is relatively smaller than that of FCSOC, showing a better tracking performance.

Figure 11 shows the angular velocity and acceleration curves of the IFCSOC controller. The chattering of velocity and acceleration is reasonable. The proposed control method can be applied to the shipborne rocket launcher system. 


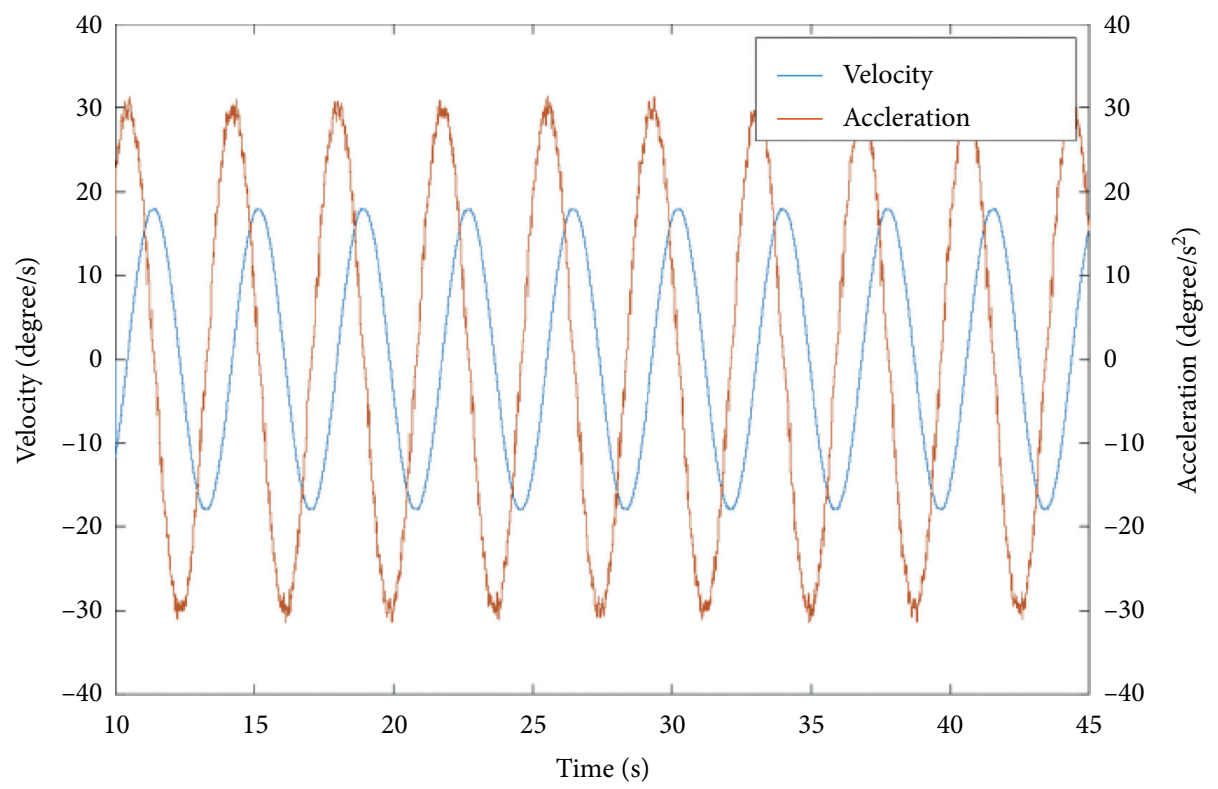

FIGURE 11: Velocity and acceleration curves.

\section{Conclusion}

The proposed IFCSOC method can deal with the nonlinearities and rapidly changing disturbances caused by complex sea conditions of the rocket launcher system. Three comparative simulations are carried out, showing that the new method can improve robustness and tracking accuracy of the system. Finally, the semiphysical experiment validates the effectiveness of IFCSOC strategy.

\section{Data Availability}

The data used to support the findings of this study are available from the corresponding author upon request.

\section{Conflicts of Interest}

The authors declare no conflicts of interest.

\section{Acknowledgments}

This research was funded by the National Natural Science Foundation of China under Grant 51805264 and the Postgraduate Research \& Practice Innovation Program of Jiangsu Province under Grant KYCX19_0266.

\section{References}

[1] S. Li, H. Liu, and S. Ding, "A speed control for a PMSM using finite-time feedback control and disturbance compensation," Transactions of the Institute of Measurement and Control, vol. 32, no. 2, pp. 170-187, 2010.

[2] Y. Yuan, Z. Yu, and L. Guo, "A sampled-data approach to nonlinear ESO-based active disturbance rejection control for pneumatic muscle actuator systems with actuator saturations," IEEE Transactions on Industrial Electronics, vol. 66, no. 6, pp. 4608-4617, 2019.
[3] K. Zhao, T. Yin, C. Zhang et al., "Robust model-free nonsingular terminal sliding mode control for PMSM demagnetization fault,” IEEE Access, vol. 7, pp. 15737-15748, 2019.

[4] H. Du, C. Qian, S. Li, and Z. Chu, "Global sampled-data output feedback stabilization for a class of uncertain nonlinear systems," Automatica, vol. 99, pp. 403-411, 2019.

[5] C. Xia, T. Liu, T. Shi, and Z. Song, "A simplified finite-controlset model-predictive control for power converters," IEEE Transactions on Industrial Informatics, vol. 10, no. 2, pp. 991-1002, 2014.

[6] Y.-J. Wu and G.-F. Li, "Adaptive disturbance compensation finite control set optimal control for PMSM systems based on sliding mode extended state observer," Mechanical Systems and Signal Processing, vol. 98, pp. 402-414, 2018.

[7] J. Jin and S. Ge, "Finite-time active disturbance rejection control based on high-order sliding mode," in Proceedings of the 2019 IEEE International Conference on Mechatronics and Automation (ICMA), IEEE, Tianjin, China, August 2019.

[8] L. Hou, Y. Ren, and Y. Xu, "SM-ADRC of permanent magnet synchronous motor based on NESO," Control Engineering of China, vol. 26, no. 1, pp. 50-54, 2019, in Chinese.

[9] B.-Z. Guo and Z.-L. Zhao, "On convergence of tracking differentiator," International Journal of Control, vol. 84, no. 4, pp. 693-701, 2011.

[10] B.-Z. Guo and Z.-L. Zhao, "Weak convergence of nonlinear high-gain tracking differentiator," IEEE Transactions on Automatic Control, vol. 58, no. 4, pp. 1074-1080, 2013.

[11] D. Tian, H. Shen, and M. Dai, "Improving the rapidity of nonlinear tracking differentiator via feedforward," IEEE Transactions on Industrial Electronics, vol. 61, no. 7, pp. 3736-3743, 2014.

[12] X. Wang and B. Shirinzadeh, "High-order nonlinear differentiator and application to aircraft control," Mechanical Systems and Signal Processing, vol. 46, no. 2, pp. 227-252, 2014.

[13] W. Wang, "The novel reconstruction strategy for signals with uncertain dynamic properties," in Proceedings of the 2011 4th International Congress on Image and Signal Processing, pp. 2499-2503, Shanghai, China, October 2011. 
[14] J. Feng, W. Wang, and Y. Chen, "An improved trackingdifferentiator filter based on Taylor's formula," Optik, vol. 158, pp. 1026-1033, 2018.

[15] W.-H. Chen, J. Yang, L. Guo, and S. Li, "Disturbance-observer-based control and related methods-an overview," IEEE Transactions on Industrial Electronics, vol. 63, no. 2, pp. 1083-1095, 2016.

[16] Z. Xu, T. Zhang, Y. Bao, H. Zhang, and C. Gerada, "A nonlinear extended state observer for rotor position and speed estimation for sensorless IPMSM drives," IEEE Transactions on Power Electronics, vol. 35, no. 1, pp. 733-743, 2020.

[17] Y. Hui, R. Chi, B. Huang, and Z. Hou, "Extended state observer-based data-driven iterative learning control for permanent magnet linear motor with initial shifts and disturbances," IEEE Transactions on Systems, Man, and Cybernetics: Systems, vol. 99, pp. 1-11, 2019.

[18] L. Zhao, Q. Li, B. Liu, and H. Cheng, "Trajectory tracking control of a one degree of freedom manipulator based on a switched sliding mode controller with a novel extended state observer framework," IEEE Transactions on Systems, Man, and Cybernetics: Systems, vol. 49, no. 6, pp. 1110-1118, 2019.

[19] W. Xu, A. K. Junejo, Y. Liu, and M. R. Islam, "Improved continuous fast terminal sliding mode control with extended state observer for speed regulation of PMSM drive system," IEEE Transactions on Vehicular Technology, vol. 68, no. 11, pp. 10465-10476, 2019.

[20] S. Kamal, A. Chalanga, M. K. Bera, and B. Bandyopadhyay, "State estimation and non vanishing unmatched disturbance reconstruction using modified super twisting algorithm," in Proceedings of the 7th International Conference on Electrical and Computer Engineering, pp. 941-944, Dhaka, Bangladesh, December 2012.

[21] G. Mohamed, A. A. Sofiane, and L. Nicolas, "Adaptive super twisting extended state observer based sliding mode control for diesel engine air path subject to matched and unmatched disturbance," Mathematics and Computers in Simulation, vol. 151, pp. 111-130, 2018.

[22] J. A. Moreno, "A linear framework for the robust stability analysis of a generalized super-twisting algorithm," in Proceedings of the 2009 6th International Conference on Electrical Engineering, Computing Science and Automatic Control (CCE), pp. 1-6, Toluca, Mexico, January 2009.

[23] L. Zhao, B. Zhang, H. Yang, and Y. Wang, "Finite-time tracking control for pneumatic servo system via extended state observer," IET Control Theory \& Applications, vol. 11, no. 16, pp. 2808-2816, 2017.

[24] L. Q. Wu, H. Lin, and J. Q. Han, "Study of tracking-differentiator on filtering," Journal of System Simulation, vol. 16, no. 670, pp. 651-652, 2004, in Chinese. 